\title{
A Recent Update of Clinical and Research Topics Concerning Adult Moyamoya Disease
}

\author{
Jin Pyeong Jeon, M.D., ${ }^{1}$ Jeong Eun Kim, M.D., Ph.D. ${ }^{2}$ \\ Department of Neurosurgery, ${ }^{1}$ Hallym University College of Medicine, Chuncheon, Korea \\ Department of Neurosurgery, ${ }^{2}$ Seoul National University College of Medicine, Seoul National University Hospital, Seoul, Korea
}

A better understanding of moyamoya disease (MMD), such as natural clinical course, surgical outcomes and research, has been obtained. This review article focuses on an giving an update for adult MMD in the Korean population. In this paper, we mainly discuss the results of our domestic investigations including meta-analysis, and related subjects from other countries.

Key Words : Moyamoya disease $\cdot$ Hemorrhage $\cdot$ Ischemia $\cdot$ Korea.

\section{INTRODUCTION}

Moyamoya disease (MMD), defined as a chronic progressive steno-occlusive disorder at the distal internal carotid artery (ICA) with abnormal collateral vessels ${ }^{55}$, has been increasingly detected, due to the advances in radiological testing and health check-up systems ${ }^{17)}$. The estimated prevalence of MMD in Japan increased from 3900 in 1994 to 7700 cases in $2003^{37)}$. Chen et al. ${ }^{4)}$ reported an annual incidence of 0.15 per 100000 people, during 2000 to 2011 in Taiwan. In particular, the incidence rate ratio of adult MMD patients between 2010 to 2011 was 1.74, with the incidence between 2000 and 2001 as a reference value. In Korea, the crude prevalence rate of MMD has increased from 6.6 in 2005 to 19.5 in $2013 .^{35)}$ The average of annual increase of prevalence was estimated to $22.3 \%$. The female incidence of MMD has increased from 3.2 in 2005 to 5.7 in $2013^{35)}$. Ahn et al. ${ }^{1)}$ examined the nationwide clinicoepidemiological features of MMD in Korea, using data from National Health Insurance from 2007 to 2011. A total of 8154 patients were diagnosed with MMD. The average age was 36.8 years, and the female-to-male ratio was 1.8. The bimodal age pattern was noted, with a first peak at age 1019 years, and a second peak at age 50-59 years. The annual incidence of MMD increased from 1.7 in 2007, to 2.3 cases per 100000 persons in 2011. For adult MMD, 1 and 5-year survival rates were $96.9 \%$, and $92.9 \%$, respectively.

Alongside the advances of radiological technology for increased identification of MMD, the results of clinical outcome, surgical outcome and research to understand MMD pathogenesis, have also been increasingly reported, further improving diagnosis capabilities. This review article focuses on an giving an update for adult MMD in the Korean population, specifically in terms of clinical course, treatment outcome and researches. In this paper, we mainly discuss the results of our domestic investigations including meta-analysis, and related subjects from other countries.

\section{THE NATURAL COURSE OF ADULT MMD}

Kuroda et al. ${ }^{39)}$ investigated the incidence and clinical features of MMD progression in an adult population, during a mean follow-up of 73.6 months. MMD progression was noted 15 of the 63 patients $(23.8 \%)$ studied. More specifically, disease progression comprised of 4 cases (36.4\%) of unilateral MMD and 11 cases $(21.2 \%)$ of bilateral MMD. The mean time to progression from diagnosis was 60.0 months (ranging from 1.5 to 8 years). Female gender was a risk factor for future MMD progression. Gross et al. ${ }^{11)}$ reviewed 42 cases of North American adults with MMD (bilateral MMD, n=19; unilateral MMD, n=17) and moyamoya syndrome $(n=6)$. The overall annual stroke rate was $13.3 \%$, and the annual hemorrhage rate was $1.7 \%$. Female gen$\operatorname{der}(p=0.031)$ and recent stroke events (within 3 years) $(p=0.035)$ were significantly associated with future stroke events. For asymptomatic patients, a nation-wide survey ${ }^{38)}$ revealed that 7 cases of

- Received : February 24, 2016 • Revised : June 2, 2016 • Accepted : July 20, 2016

- Address for reprints : Jeong Eun Kim, M.D., Ph.D.

Department of Neurosurgery, Seoul National University College of Medicine, Seoul National University Hospital, 101 Daehak-ro, Jongno-gu, Seoul 03080, Korea

Tel : +82-2-2072-2354, Fax : +82-2-744-8459, E-mail : eunkim@snu.ac.kr

- This is an Open Access article distributed under the terms of the Creative Commons Attribution Non-Commercial License (http://creativecommons.org/licenses/by-nc/3.0) which permits unrestricted non-commercial use, distribution, and reproduction in any medium, provided the original work is properly cited. 
future stroke (transient ischemic attack, $n=3$; ischemic stroke, $\mathrm{n}=1$; hemorrhage stroke, $\mathrm{n}=3$ ) were observed during a mean follow-up time of 43.7 months. MMD progression was related to silent infarction or ischemic events. As such, they concluded that asymptomatic MMD had a dynamic nature, with an estimated annual stoke risk rate of 3.2\%. Importantly, however, the difference in the severity of hemodynamic status, and the presence of symptoms, can limit the interpretation of the previous results. Cho et al. ${ }^{5)}$ examined the natural clinical course of adult MMD patients $(n=241)$ who were hemodynamically stable during a mean follow-up times of 82.5 months. The overall annual stoke rate was $4.5 \%$ per person-year. The hemorrhage presenting group showed a $4.3 \%$ rate of annual hemorrhage stroke, and the ischemic presenting group showed a 3.0\% rate of annual ischemic stroke. For asymptomatic MMD patients, with hemodynamically stable status, an overall annual stroke rate of 3.4\% ( $2.5 \%$ of hemorrhage stroke and $0.8 \%$ of ischemic stroke) was observed. The presence of familial MMD [hazord ratio (HR), $2.62, p=0.009$ ], and thyroid disease (HR, 2.56, $p=0.02)$ were risk factors for overall stroke.

Another issue is a clinical course of unilateral MMD, in particular contralateral angiographic progression. The incidence rate of contralateral progression in unilateral $\mathrm{MMD}$, has been reported, ranging from $0-50 \%{ }^{13,53)}$. The presence of contralateral abnormality, as well as female gender, have been suggested as risk factors for future progression ${ }^{28,39)}$. A literature review revealed that only two studies analyzed progression rates and the associated factors in adult unilateral $\mathrm{MMD}^{13,39)}$. Kuroda et al. ${ }^{39)}$ found that $36.4 \%$ ( 4 out of 11 cases) showed a contralateral progression rate during a mean follow-up of 20 months. They suggested female gender as a risk factor for this contralateral progression. In contrast, Hallemeier et al. ${ }^{13)}$ did not find any risk factor for future progression in 12 adult unilateral MMD patients. In spite of this, confounding variables, such as heterogeneity of the treatment methods used (medical or surgical treatments), and small numbers of the enrolled patients ( $n=11$ or 12 ) were concerns when interpreting the results in the adult MMD cases. Consequently, we wonder how high the contralateral progression rate is in adult MMD patients following surgical revascularization $^{31}$. Six patients (14.6\%) experienced contralateral progression during a mean follow-up period of 34 months (range 21-64 months) in our cohort. Angiographic abnormalities, defined as equivocal or mild A1 segment of the anterior cerebral artery (ACA), M1 segment of the middle cerebral artery (MCA) and ICA stenosis on angiography ${ }^{23,28)}$ was significantly associated with future progression (odds ratio, 49.00; $p=0.04$ ) ${ }^{41)}$. Accordingly, unilateral MMD cases with contralateral angiographic abnormalities, should be monitored closely.

\section{SURGICAL TREATMENT OUTCOMES}

Revascularization surgery has shown to have a beneficial effect : reducing ischemic stroke and improving cerebral hemo- dynamics $^{31)}$. Guzman et al. ${ }^{12)}$ reported the treatment outcomes of 233 adult MMD, and 96 pediatric MMD patients. Direct bypass was performed in $95.1 \%$ of adults, and $76.2 \%$ of pediatric MMD patients. Fifteen cases of significant neurologic deficits (ischemic events : $\mathrm{n}=8$; hemorrhagic events, $\mathrm{n}=7$ ) were noted. The cumulative 5-year risk of post-operative, subsequent death or stroke was estimated to be $5.5 \%$. They concluded that revascularization surgery was effective for the prevention of future ischemic stroke. Mesiwala et al. ${ }^{45)}$ also reported the treatment outcomes of $39 \mathrm{MMD}$ patients (Caucasian, $\mathrm{n}=27$; Asian, $\mathrm{n}=12$ ) from the Western USA. In their study, direct bypass was performed in 36 patients with 59 surgical procedures, and indirect bypass of encephalo-duro-arterio-synangiosis (EDAS) in 3 patients with 6 procedures. The procedure-related mortality rate was $4.61 \%$. They concluded that revascularization surgery may lower the risk of future ischemic stroke. Nevertheless, two factors of clinical presentation (ischemic vs. hemorrhage) and surgical techniques (direct vs. indirect), should be considered when assessing treatment outcomes in adult MMD patients. Hemorrhage presenting MMD adult patients showed a more advanced angiographic status and more pseudoaneurysms than ischemic presenting MMD adult patients ${ }^{19)}$. Jang et al. ${ }^{19)}$ investigated the differences in major artery occlusion and collateral vessels, according to their presentation. Adult MMD patients with hemorrhage presentation showed a higher frequency of major artery occlusion and collateral formation, than those from the ischemic presentation. In particular, MMD patients with deep-seated intracranial hemorrhage $(\mathrm{ICH})$ revealed a significantly higher ACA occlusion rate, than those with lobar ICH. Liu et al. ${ }^{44)}$ also reported that extensive dilatation of the anterior choroidal and posterior communicating arteries were related to hemorrhage development in adult MMD patients, especially intraventricular hemorrhage. Accordingly, treatment outcome should be differently assessed, according to presentation, due to the different associated angiographic architectures. The surgical benefits of revascularization surgery for preventing future hemorrhage remains controversial, due to a relatively small number of the enrolled patients, and the retrospective nature of the previous studies. Kawaguchi et al. ${ }^{27)}$ reported that future stroke events (rebleeding or ischemic events) between two groups of patients with direct bypass (none of 6 cases) and patients with conservative (6 of 11 cases) or EDAS (3 of 5 cases) did differ significantly. Fujii et al. ${ }^{9}$ did not find efficacy of direct bypass ( $n=29,19.1 \%)$ affected future rebleeding events in hemorrhagic MMD patients, compared to those in the conservative group $(n=39,28.3 \%)$. In terms of the angiographic outcome, direct bypass was highly effective (42 of 47 cases, 90\%), compared with indirect bypass (18 of 47 cases, $38 \%)^{16)}$. The Japan Adult Moyamoya (JAM) trial ${ }^{46)}$ investigated the surgical benefits of reducing rebleeding events and patients' prognosis. Rebleeding events were noted in 5 patients in the surgical group (11.9\%) and in 12 patients in the non-surgical group (31.6\%) $(p=0.052)$. Since the Kaplan-Meier survival analysis revealed a statistically marginal difference between the surgical and 
non-surgical groups ( $p=0.042)$, the JAM trial suggested that a direct bypass has a preventative effect on the recurrence of future hemorrhage in the adult MMD population.

Kim et al. ${ }^{29)}$ investigated treatment outcomes according to surgical methods [combined superficial temporal artery-middle cerebral artery (STA-MCA) anastomosis with encephalo-duroarterio-galeo-synangiosis (EDAGS) vs. EDAGS with or without inversion] in adult MMD patients with non-hemorrhagic presentation. Combined bypass showed a higher angiographic revascularization area than direct bypass. Peri-operative complications were found more frequently in patients treated with combined bypass, but the clinical outcomes did not differ significantly according to the surgical methods used. They concluded that EDAGS can be a reliable alternative to combined bypass in adult MMD cases, however, they did not provide detailed information on the future stroke rate according to surgical method. Choi et al. . $^{7}$ showed an improved revascularization angiographic outcome of combined bypass, than from indirect bypass, in adult MMD patients. In their study, a revascularization area of more than two-thirds of the MCA distribution was more frequently observed in the combined bypass group ( $n=12,48.0 \%)$, than the indirect bypass group $(n=9,27.3 \%)$. Revascularization pattern in adult patients ( $\geq 18$ years old) who underwent STAMCA anastomosis with encephalo-moy-synongiosis (EMS), showed more cases of EMS dominance $(n=8,32.0 \%)$ or an equivalent contribution to revascularization $(n=9,36.0 \%)^{18)}$.

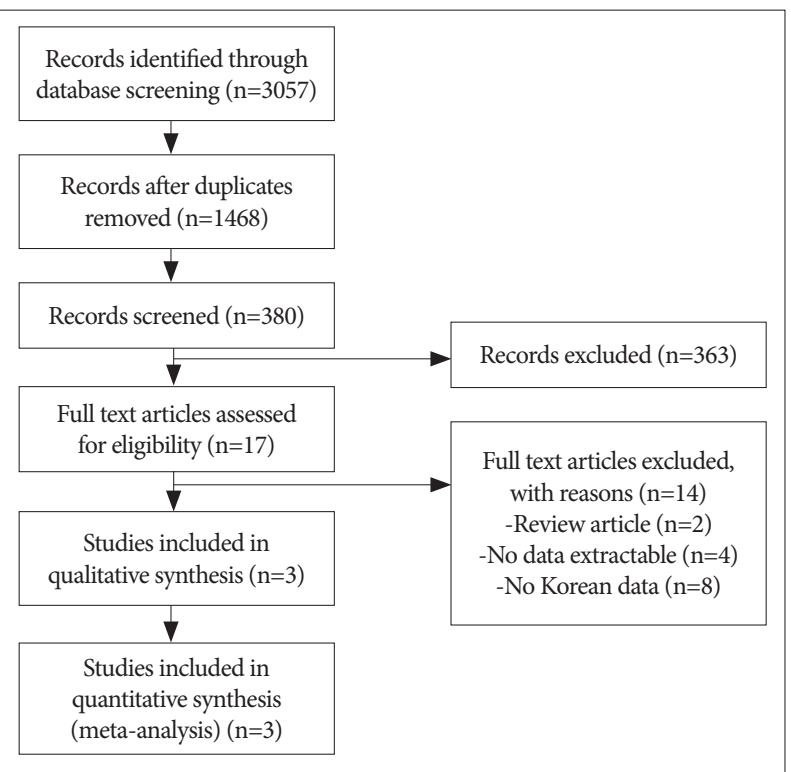

Fig. 1. Flow diagram for identification of relevant studies.
Regarding cases undergoing EDAS with EMS, most cases ( $\mathrm{n}=27$, $96.4 \%)$ revealed EMS dominance $(n=14,50.0 \%)$ or an equivalent contribution to revascularization ( $n=13,46.4 \%)$. Accordingly, they concluded that EMS plays a key role in each combined surgical technique ${ }^{18)}$. In Korea, Cho et al. ${ }^{6}$ reported the long-term treatment outcomes of combined STA-MCA anastomosis with EDGS, in adult MMD patients. The clinical status continued to improve postoperatively, and plateaued 6 months after surgery. The bypass patency at immediate post-operative (within 2 weeks after procedures), short-term (5-12 months after procedures) and long-term (over 51 months after procedure) were $96.1 \%, 95.8 \%$ and $80.6 \%$, respectively. The revascularization area, defined as the perfusion area/supratentorial area $\times 100$, increased from $44.2 \pm 15.9 \%$ in the short-term to $54.8 \pm 18.7 \%$ in the long-term follow-up period. Mean cerebral blood flow in the MCA territory increased in the short-term period and plateaued. Three cases of stroke, two hemorrhagic and one ischemic event, were noted during a mean follow-up period of 71.0 months. The annual rate of stroke in the operated hemisphere was as follows : symptomatic hemorrhage rate, $0.4 \%$ /personyear; infarction rate, $0.2 \%$ /person-year. The 5 -year event-free survival rates of symptomatic hemorrhage and cerebral infarction ipsilateral to the operated side, were all 98.7\%.

\section{SURGICAL EFFICAY IN SYMPTOMATIC ADULT MMD IN KOREA : A META-ANALYSIS}

We conducted a meta-analysis to evaluate treatment outcomes of secondary stroke prevention after surgical treatments in Korean adult MMD patients. Detailed information on the searching, inclusion criteria, and statistics is described in supplemental data. A flow diagram of the detailed search process was described in Fig. 1. After records screening and deciding eligibility, 3 articles were included finally (Table 1$)^{8,36,40)}$. A total of 627 patients from 3 studies were included to assess surgical efficacy in preventing recurrent stroke compared to conservative treatment (Fig. 2A). Among them, 460 patients underwent 501 cases of bypass surgery and 167 did conservative treatment. Bypass surgery showed a significant prevention effect of recurrent stroke compared to conservative treatments in adult MMD (OR, 0.335; 95\% CI : 0.198-0.566, $p<0.001$ ). Regarding publication bias, Egger's regression test showed -1.17 of intercept [ $(5 \%$ CI, -44.32 to $41.97 ; \mathrm{t}=0.35, \mathrm{df}=1$; and $p=0.78$ ( 2 tailed)]. For the rank correlation test, Kendall's tau was -0.33 with 2 -tailed $p=0.60$. Accordingly, there was no evidence of publication bias in this comparison (Fig. 2B).

Table 1. Clinical data of the studies included in this meta-analysis

\begin{tabular}{|c|c|c|c|c|}
\hline Author (year) & Study design & Treatment & Sample size (n) & NOS score \\
\hline Lee et al. ${ }^{40)}(2012)$ & Retrospective & Surgery, conservative & 142 & 6 \\
\hline Choi et al. ${ }^{8)}$ (2013) & Retrospective & Surgery, conservative & 44 & 6 \\
\hline Kim et al. ${ }^{36)}(2015)$ & Retrospective & Surgery, conservative & 441 & 7 \\
\hline
\end{tabular}




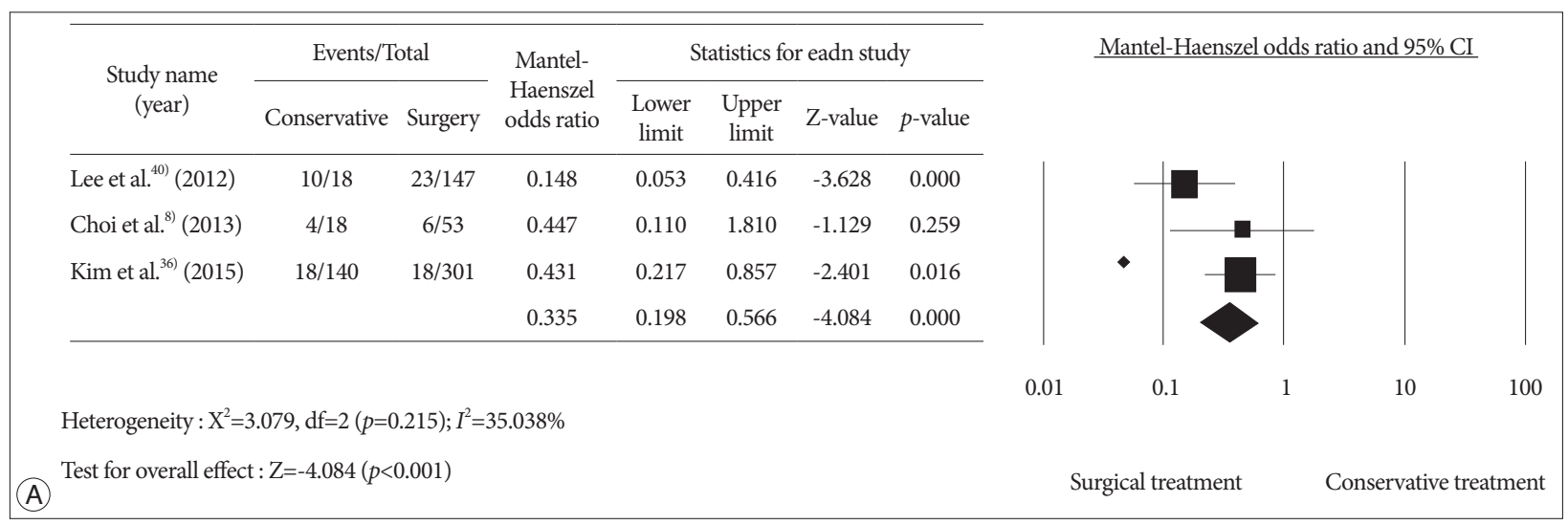

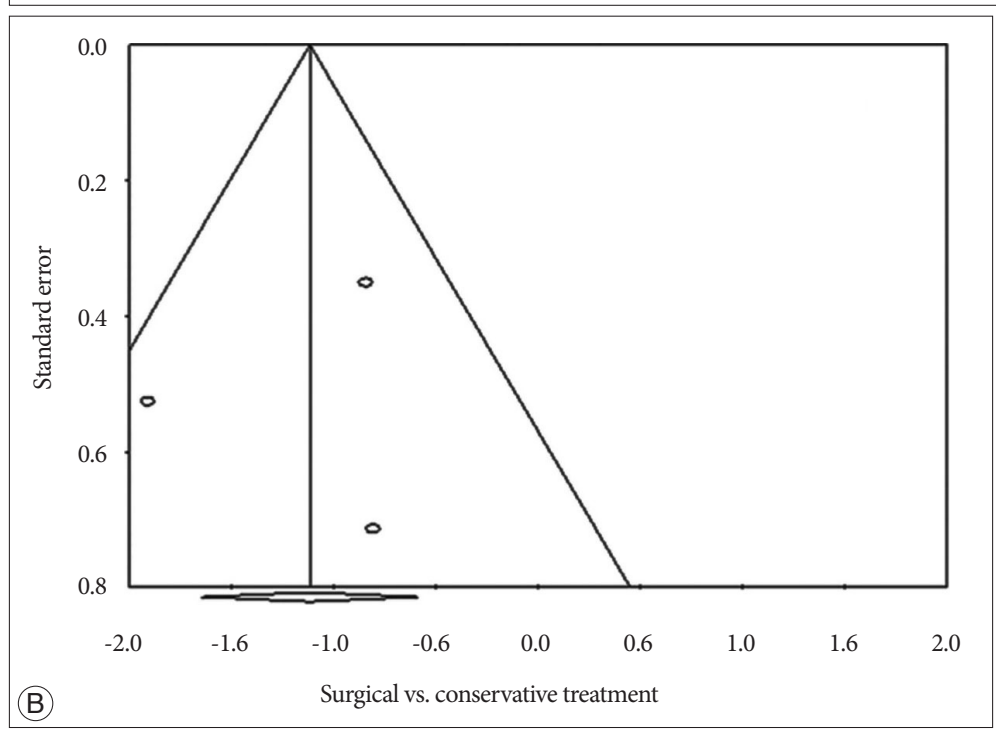

Fig. 2. Forest plot for recurrent stroke events according to treatment methods (conservative vs. surgery) (A). Funnel plots for publication bias according to treatment methods (B).
Recently, two meta-analysis of the MMD treatment outcome have been published ${ }^{51,54)}$. Qian et al. $^{51)}$ reported that bypass surgery significantly reduced future stroke than conservative treatment (OR 0.17; 95\% CI : 0.12-0.26, $p<0.01$ ). But they did not find surgical benefit for MMD patients with ischemic presentation (OR, 0.45; 95\% CI : 0.15-1.29, $p=0.14$ ). Sun et al. ${ }^{54)}$ suggested that direct bypass provide better risk reduction of long-term ischemia than indirect (OR, $0.51 ; 95 \% \mathrm{CI}: 0.33-0.77)$ or combined bypass (OR, 0.47; 95\% CI : 0.31-0.72) in adult MMD. However, heterogeneous population (mixed pediatric and adult MMD patients) $)^{51)}$ and a potentially confounding factors (mixed symptomatic and asymptomatic patients) ${ }^{54)}$ were concerns to interpret the results. In this meta-analysis, we only included for Koran MMD patients. Nevertheless, small number of the studies and their retrospective nature can be limitation.

\section{RESEARCH IN MOYAMOYA DISEASE}

From genetics to metabolomics, studies of pathomechanism underlying MMD, have been increasingly reported. Most investigations have been conducted in mixed adult and pediatric MMD patients. Recently, two review articles for vascular pro- genitor cells ${ }^{26)}$ and MMD biomarkers ${ }^{52)}$ have been published in Journal of Korean Neurosurgical Society. To avoid duplication, we have focused domestic researches, except for studies of endothelial progenitor cells and smooth-muscle progenitor cells.

Human leukocyte antigen (HLA) genotyping, genome-wide linkage analysis, and genome wide association study have been conducted to find MMD-specific genetic marker. The HLA-B35 allele was significantly noted in Korean MMD patients, in particular female, late-onset MMD (defined as patients who were detected over 10 years old ${ }^{14}$. The authors suggested that HLAB35 might have a role in MMD pathogenesis in terms of autoimmunity or infection. Hong et al. ${ }^{15)}$ reported that familial MMD showed significantly increased HLA-DRB1 $\left(^{*}\right) 1302(70.0 \%)$ and DQB1 $\left(^{*} 0609\right.$ (40.0\%) phenotype frequencies, compared to non-familial MMD or the healthy control group. They suggested that HLA-DRB1 $\left(^{*}\right) 1302$ and DQB1 $\left(^{*}\right) 0609$ haplotypes could be related to intimal fibrosis, and arterial occlusion.

The association of single nucleotide polymorphisms of the tissue inhibitor of metalloproteinase (TIMP)-2 and TIMP-4 genes and MMD development were studied ${ }^{25)}$. A G/C heterozygous genotype at position -418 of TIMP-2 promoter region was observed in familial MMD (9 of 11 cases, $81.8 \%$ ), more signifi- 
cantly than that of non-familial MMD (16 of 50 cases, $32.0 \%$ ) or control groups (14 of 50 cases, $28.0 \%$ ). A G/C heterozygous genotype at position -418 of the TIMP-2 promoter region might be a genetic predisposing factor for familial MMD, through influencing Sp1 binding and subsequent TIMP-2 transcription. However, the conclusions of Kang et al. ${ }^{25)}$ was not supported by others. ${ }^{49)}$

Park et al. ${ }^{50)}$ hypothesized that genetic variation of endothelial nitric oxide synthase (eNOS) could be related to age-specific MMD. Overall, there were no differences found in eNOS polymorphisms, such as $-922 \mathrm{~A}>\mathrm{G},-786 \mathrm{~T}>\mathrm{C}$, intron 4 and $894 \mathrm{G}>\mathrm{T}$, between MMD patients and the control group. More specifically, adult MMD ( $\geq 20$ years) revealed significantly decreased $4 \mathrm{a} 4 \mathrm{~b}$ sequences, compared to the control group $(n=2,4.7 \%$ in adult MMD vs. $n=46,20.3 \%$ in control group; $p=0.029$ ). The A- $4 b-G$ haplotype was frequently observed in adult $\mathrm{MMD}$, compared to the control group. However, pediatric MMD patients ( $<20$ years) did not show significant differences in $4 \mathrm{a} 4 \mathrm{~b}$ sequences and A-4b-G haplotypes, when compared to the control group.

Ringer finger protein 213 (RNF213) has been identified as a susceptible gene for $\mathrm{MMD}^{10,43,47)}$. Kim et al. ${ }^{30)}$ reported an RNF213 genotype, and its association with clinical features in Korean MMD patients. A total of 125 (75.8\%) MMD patients examined had the c.14429G $>$ A (p.R4810K) variant. The homozygous c.14429G $>$ A (p.R4810K) RNF213 variant was significantly associated with early-onset MMD ( $<5$ years), cerebral infarction, and cognitive impairment. The RNF213 variant could also be used for MMD diagnosis in adult patients. Angiography-based MMD diagnosis can be insufficient to distinguish adult MMD from intracranial atherosclerotic stenosis (ICAS) in daily practice ${ }^{2)}$. First, the early state of MMD can be diagnosed as ICAS due to the absence of tandem stenosis of the proximal MCA, or ACA concomitant with distal ICA stenosis. Second, adult MMD tends to show less prominent basal collateral vessels than pediatric MMD. Third, atherosclerosis (dyslipidemia, heavy smoking, alcoholics and hypertension), prothrombotic conditions (head trauma and cranial radiation), and diabetes mellitus (DM) can cause steno-occlusive intracranial lesions mimicking moyamoya syndrome ${ }^{2,32)}$. Accordingly, actual incidence of adult MMD can be potentially underestimated. Bang et al. ${ }^{2)}$ analyzed the occurrence of the p.Arg4810Lys variant of RNF213 in adult patients showing intracranial stenosis. Three variables such as basal collateral vessels, bilateral involvement, and the absence of DM, were significantly correlated with the RNF213 variant. Threefourths $(75.6 \%)$ of the MMD patients $(n=131)$ who met these three variables, showed the RNF213 variant. However, the RNF213 variant was also noted in patients who met two of the criteria (57.7\%), one of the criteria (28.6\%), or no criteria (20.0\%) for MMD. Consequently, they concluded that some of the MMD patients had been misclassified as ICAS in clinical circumstances. In such complicated cases, the presence of the RNF213 variant could be very helpful for MMD detection, in particular for early state of MMD.

Disruption of balance between matrix metalloproteinases
(MMP) and TIMP which is an inhibitor for MMP could lead to intimal hyperplasia through excessive smooth muscle cell (SMC) migration and proliferation ${ }^{24) .}$ Kang et al. ${ }^{24)}$ demonstrated significantly increased MMP-9, monocyte chemoattractant protein-1 (MCP-1), interleukins-1ß, vascular endothelial growth factor (VEGF) and platelet-derived growth factor $\mathrm{BB}$, as well as decreased TIMP-1 and TIMP-2, in MMD patients, compared to those in the control groups. MCP1 and VEGF could be related to the recruitment of vascular progenitor cells and, subsequent MMD vessel formation $^{24)}$.

Elevated cellular retinoic acid-binding protein-I (CRABP-I) in the cerebrospinal fluid (CSF) has been suggested as a candidate protein for $\mathrm{MMD}^{22,34)}$. Kim et al. ${ }^{34)}$ identified a higher level of CRABP-I expression in pediatric MMD, using Western blotting. They hypothesized that CRABP-I inhibited retinoid activity, by increasing retinoic acid production, which results in neointimal thickening. Jeon et al. ${ }^{22)}$ further examined the difference in CRABP-I expression, according to diseases entity and its association with clinical phenotypes, as well as post-operative hemodynamic changes in the adult population. Bilateral MMD cases [median (25th-75th percentile); 1.45 (0.86-2.52)] had significantly higher levels of CRABP-I intensities, compared to unilateral MMD cases [0.91 (0.78-1.20)], or atherosclerotic cerebrovascular disease $[0.85(0.66-1.11)]$. CRABP-I was related to a decrease in basal collateral vessels, after surgical revascularization. CRABP-I did not differ significantly according to initial presentation (hemorrhage vs. infarction), or improvement of the vascular reserve. The authors concluded that elevated CRABP-I in the CSF can be associated with typical bilateral adult MMD.

Metabolomics has been an emerging technology in the investigation of disease-specific metabolomic patterns and biomarkers $^{20,48)}$. Jeon et al. ${ }^{21)}$ compared the CSF metabolites of adult bilateral MMD cases than those of unilateral MMD and atherosclerotic cenebrovascular disease, using hydrogen-1 nuclear magnetic resonance spectroscopy. The results showed that bilateral MMD revealed higher levels of glutamine $(p<0.001)$ and taurine $(p=0.004)$, than did atherosclerotic stenosis. Würtz et al. ${ }^{57)}$ reported that a higher glutamine level was related to intima-media thickness of the carotid arteries and coronary artery disease. Accordingly, they suspected that higher glutamine in MMD could be related to increased abnormality of SMC proliferation, and thickened intima, compared to that of atherosclerotic stenosis, although the precise mechanism is not well understood ${ }^{21)}$. Regarding bilateral and unilateral MMDs, no differences were found at the metabolite level, in their study.

Association of the MMD and thyroid diseases has been reported $^{33,42,56)}$. Kim et al. ${ }^{33)}$ investigated the relationship between thyroid autoantibodies and MMD ( $\mathrm{n}=63)$ under a euthyroid state through a comparative study with non-MMD stroke $(n=71)$ or healthy controls $(n=200)$. In their study, thyroid autoantibodies were found to be significantly higher in MMD patients, than in the control group. They concluded that immune aberrancies related to thyroid autoimmunity may have a role in MMD patho- 
genesis. T-cell dysregulation $^{56)}$ or increased sensitivity to the sympathetic nervous system of the vessel ${ }^{42)}$ was thought to be responsible for abnormal SMCs proliferation and moyamoya vessel formation. Recently, Chen et al. ${ }^{3)}$ analyzed the differences of the autoimmune diseases prevalence between unilateral $[n=68$, $38.9 \pm 11.5$ years (age at onset, mean \pm SD)] and bilateral MMD $(\mathrm{n}=316,37.3 \pm 13.7$ years). Although specific autoimmune disease, such as thyroid disease, type 1 DM or systemic lupus erythematosus did not differ significantly in their prevalence, overall autoimmune disease was observed to be significantly higher unilateral MMD, compared to bilateral MMD [n=18 (26.5\%) in unilateral MMD vs. $\mathrm{n}=43$ (13.6\%) in bilateral MMD; $p=0.008)]$. Nevertheless, the actual pathogenic mechanisms of the autoimmune disease in the development of MMD are still poorly understood. Accordingly, studies concerning the autoimmune mechanism in MMD development, in particular the role of elevated thyroid autoantibodies in MMD development and progression, and its therapeutic targets, are required ${ }^{31,33)}$.

\section{CONCLUSION}

A better understanding of MMD, including its natural clinical course, treatment outcomes and research has been obtained; nevertheless, most previous studies reveal non-concordant results, due to heterogeneity of the population (pediatric and adult), hemodynamic status (stable or unstable), disease entities (MMD only or mixed with moyamoya syndrome), treatment methods (direct bypass, indirect bypass, or combined bypass). Future adult MMD studies and treatments should be approached using updated knowledge, for instance using multicenter, randomized studies to evaluate the clinical course and surgical outcome, as well as research to identify MMD biomarkers related to clinical phenotypes, such as future stroke prediction and disease progression without including pediatric MMD patients.

\section{- Acknowledgements}

This study was supported by BioGreen 21 (PJ009051) of Rural Development Administration. We would like to thank Sung-Eun Kim for help with the data collection.

\section{References}

1. Ahn IM, Park DH, Hann HJ, Kim KH, Kim HJ, Ahn HS : Incidence, prevalence, and survival of moyamoya disease in Korea : a nationwide, population-based study. Stroke 45 : 1090-1095, 2014

2. Bang OY, Ryoo S, Kim SJ, Yoon CH, Cha J, Yeon JY, et al. : Adult moyamoya disease : a burden of intracranial stenosis in east asians? PLoS One 10 : e 0130663,2015

3. Chen JB, Liu Y, Zhou LX, Sun H, He M, You C : Increased prevalence of autoimmune disease in patients with unilateral compared with bilateral moyamoya disease. J Neurosurg $124: 1215-1220,2016$

4. Chen PC, Yang SH, Chien KL, Tsai IJ, Kuo MF : Epidemiology of moyamoya disease in Taiwan : a nationwide population-based study. Stroke $45: 1258-1263,2014$

5. Cho WS, Chung YS, Kim JE, Jeon JP, Son YJ, Bang JS, et al. : The natural clinical course of hemodynamically stable adult moyamoya disease. J
Neurosurg 122 : 82-89, 2015

6. Cho WS, Kim JE, Kim CH, Ban SP, Kang HS, Son YJ, et al. : Long-term outcomes after combined revascularization surgery in adult moyamoya disease. Stroke 45 : 3025-3031, 2014

7. Choi IJ, Cho SJ, Chang JC, Park SQ, Park HK : Angiographic results of indirect and combined bypass surgery for adult moyamoya disease. $\mathrm{J}$ Cerebrovasc Endovasc Neurosurg 14 : 216-222, 2012

8. Choi WS, Lee SB, Kim DS, Huh PW, Yoo DS, Lee TG, et al. : Thirteenyear experience of 44 patients with adult hemorrhagic moyamoya disease from a single institution : clinical analysis by management modality. J Cerebrovasc Endovasc Neurosurg 15 : 191-199, 2013

9. Fujii K, Ikezaki K, Irikura K, Miyasaka Y, Fukui M : The efficacy of bypass surgery for the patients with hemorrhagic moyamoya disease. Clin Neurol Neurosurg 99 Suppl 2 : S194-S195, 1997

10. Fujimura M, Sonobe S, Nishijima Y, Niizuma K, Sakata H, Kure S, et al. : Genetics and biomarkers of moyamoya disease : significance of RNF213 as a susceptibility gene. J Stroke $16: 65-72,2014$

11. Gross BA, Du R: The natural history of moyamoya in a North American adult cohort. J Clin Neurosci 20 : 44-48, 2013

12. Guzman R, Lee M, Achrol A, Bell-Stephens T, Kelly M, Do HM, et al. : Clinical outcome after 450 revascularization procedures for moyamoya disease. Clinical article. J Neurosurg 111 : 927-935, 2009

13. Hallemeier CL, Rich KM, Grubb RL Jr, Chicoine MR, Moran CJ, Cross DT 3rd, et al. : Clinical features and outcome in North American adults with moyamoya phenomenon. Stroke 37 : 1490-1496, 2006

14. Han H, Pyo CW, Yoo DS, Huh PW, Cho KS, Kim DS : Associations of moyamoya patients with HLA class I and class II alleles in the Korean population. J Korean Med Sci $18:$ 876-880, 2003

15. Hong SH, Wang KC, Kim SK, Cho BK, Park MH : Association of HLADR and -DQ genes with familial moyamoya disease in Koreans. J Korean Neurosurg Soc $46: 558-563,2009$

16. Houkin K, Kamiyama H, Abe H, Takahashi A, Kuroda S : Surgical therapy for adult moyamoya disease. Can surgical revascularization prevent the recurrence of intracerebral hemorrhage? Stroke 27 : 1342-1346, 1996

17. Im SH, Cho CB, Joo WI, Chough CK, Park HK, Lee KJ, et al. : Prevalence and epidemiological features of moyamoya disease in Korea. J Cerebrovasc Endovasc Neurosurg 14 : 75-78, 2012

18. Imai H, Miyawaki S, Ono H, Nakatomi H, Yoshimoto Y, Saito N : The importance of encephalo-myo-synangiosis in surgical revascularization strategies for moyamoya disease in children and adults. World Neurosurg 83 : 691-699, 2015

19. Jang DK, Lee KS, Rha HK, Huh PW, Yang JH, Park IS, et al. : Clinical and angiographic features and stroke types in adult moyamoya disease. AJNR Am J Neuroradiol 35 : 1124-1131, 2014

20. Jeon JP, Kim JE : NMR-based metabolomics analysis of leptomeningeal carcinomatosis. Neurosurgery 75 : N12-N13, 2014

21. Jeon JP, Yun T, Jin X, Cho WS, Son YJ, Bang JS, et al. : 1H-NMR-based metabolomic analysis of cerebrospinal fluid from adult bilateral moyamoya disease : comparison with unilateral moyamoya disease and atherosclerotic stenosis. Medicine (Baltimore) 94 : e629, 2015

22. Jeon JS, Ahn JH, Moon YJ, Cho WS, Son YJ, Kim SK, et al. : Expression of cellular retinoic acid-binding protein-I (CRABP-I) in the cerebrospinal fluid of adult onset moyamoya disease and its association with clinical presentation and postoperative haemodynamic change. J Neurol Neurosurg Psychiatry 85 : 726-731, 2014

23. Jeon JS, Sheen SH, Hwang GJ, Kim HC, Kwon BJ : Feasibility of intravenous flat panel detector CT angiography for intracranial arterial stenosis. AJNR Am J Neuroradiol 34 : 129-134, 2013

24. Kang HS, Kim JH, Phi JH, Kim YY, Kim JE, Wang KC, et al. : Plasma matrix metalloproteinases, cytokines and angiogenic factors in moyamoya disease. J Neurol Neurosurg Psychiatry 81 : 673-678, 2010 
25. Kang HS, Kim SK, Cho BK, Kim YY, Hwang YS, Wang KC, et al. : Single nucleotide polymorphisms of tissue inhibitor of metalloproteinase genes in familial moyamoya disease. Neurosurgery 58 : 1074-1080; discussion 1074-1080, 2006

26. Kang HS, Wang KC, Kim SK : Circulating vascular progenitor cells in moyamoya disease. J Korean Neurosurg Soc 57 : 428-431, 2015

27. Kawaguchi S, Okuno S, Sakaki T : Effect of direct arterial bypass on the prevention of future stroke in patients with the hemorrhagic variety of moyamoya disease. J Neurosurg $93: 397-401,2000$

28. Kelly ME, Bell-Stephens TE, Marks MP, Do HM, Steinberg GK : Progression of unilateral moyamoya disease : a clinical series. Cerebrovasc Dis $22: 109-115,2006$

29. Kim DS, Huh PW, Kim HS, Kim IS, Choi S, Mok JH, et al. : Surgical treatment of moyamoya disease in adults : combined direct and indirect vs. indirect bypass surgery. Neurol Med Chir (Tokyo) 52 : 333-338, 2012

30. Kim EH, Yum MS, Ra YS, Park JB, Ahn JS, Kim GH, et al. : Importance of RNF213 polymorphism on clinical features and long-term outcome in moyamoya disease. J Neurosurg 124 : 1221-1227, 2016

31. Kim JE, Jeon JS : An update on the diagnosis and treatment of adult Moyamoya disease taking into consideration controversial issues. Neurol Res 36 : 407-416, 2014

32. Kim JE, Kim KM, Kim JG, Kang HS, Bang JS, Son YJ, et al. : Clinical features of adult moyamoya disease with special reference to the diagnosis. Neurol Med Chir (Tokyo) 52 : 311-317, 2012

33. Kim SJ, Heo KG, Shin HY, Bang OY, Kim GM, Chung CS, et al. : Association of thyroid autoantibodies with moyamoya-type cerebrovascular disease : a prospective study. Stroke 41 : 173-176, 2010

34. Kim SK, Yoo JI, Cho BK, Hong SJ, Kim YK, Moon JA, et al. : Elevation of CRABP-I in the cerebrospinal fluid of patients with Moyamoya disease. Stroke 34 : 2835-2841, 2003

35. Kim T, Lee H, Bang JS, Kwon OK, Hwang G, Oh CW : Epidemiology of moyamoya disease in Korea : based on national health insurance service Data. J Korean Neurosurg Soc 57 : 390-395, 2015

36. Kim T, Oh CW, Kwon OK, Hwang G, Kim JE, Kang HS, et al. : Stroke prevention by direct revascularization for patients with adult-onset moyamoya disease presenting with ischemia. J Neurosurg 124 : 1788-1793, 2016

37. Kuriyama S, Kusaka Y, Fujimura M, Wakai K, Tamakoshi A, Hashimoto S, et al. : Prevalence and clinicoepidemiological features of moyamoya disease in Japan : findings from a nationwide epidemiological survey. Stroke 39 : 42-47, 2008

38. Kuroda S, Hashimoto N, Yoshimoto T, Iwasaki Y; Research Committee on Moyamoya Disease in Japan : Radiological findings, clinical course, and outcome in asymptomatic moyamoya disease : results of multicenter survey in Japan. Stroke 38 : 1430-1435, 2007

39. Kuroda S, Ishikawa T, Houkin K, Nanba R, Hokari M, Iwasaki Y : Incidence and clinical features of disease progression in adult moyamoya disease. Stroke 36 : 2148-2153, 2005

40. Lee SB, Kim DS, Huh PW, Yoo DS, Lee TG, Cho KS : Long-term followup results in 142 adult patients with moyamoya disease according to management modality. Acta Neurochir (Wien) 154 : 1179-1187, 2012

41. Lee SC, Jeon JS, Kim JE, Chung YS, Ahn JH, Cho WS, et al. : Contralateral progression and its risk factor in surgically treated unilateral adult moyamoya disease with a review of pertinent literature. Acta Neurochir
(Wien) $156: 103-111,2014$

42. Liu JS, Juo SH, Chen WH, Chang YY, Chen SS : A case of Graves' diseases associated with intracranial moyamoya vessels and tubular stenosis of extracranial internal carotid arteries. J Formos Med Assoc 93 : 806-809, 1994

43. Liu W, Morito D, Takashima S, Mineharu Y, Kobayashi H, Hitomi T, et al. : Identification of RNF213 as a susceptibility gene for moyamoya disease and its possible role in vascular development. PLoS One 6 : e22542, 2011

44. Liu W, Zhu S, Wang X, Yue X, Zhou Z, Wang H, et al. : Evaluation of angiographic changes of the anterior choroidal and posterior communicating arteries for predicting cerebrovascular lesions in adult moyamoya disease. J Clin Neurosci 18 : 374-378, 2011

45. Mesiwala AH, Sviri G, Fatemi N, Britz GW, Newell DW : Long-term outcome of superficial temporal artery-middle cerebral artery bypass for patients with moyamoya disease in the US. Neurosurg Focus 24 : E15, 2008

46. Miyamoto S, Yoshimoto T, Hashimoto N, Okada Y, Tsuji I, Tominaga T, et al. : Effects of extracranial-intracranial bypass for patients with hemorrhagic moyamoya disease : results of the Japan Adult Moyamoya Trial. Stroke 45 : 1415-1421, 2014

47. Miyatake S, Miyake N, Touho H, Nishimura-Tadaki A, Kondo Y, Okada I, et al. : Homozygous c.14576G $>$ A variant of RNF213 predicts early-onset and severe form of moyamoya disease. Neurology 78 : 803-810, 2012

48. Nicholson JK, Lindon JC : Systems biology : metabonomics. Nature 455 : 1054-1056, 2008

49. Paez MT, Yamamoto T : Single nucleotide polymorphisms of tissue inhibitor of metalloproteinase genes in familial moyamoya disease. Neurosurgery 60 : E582; author reply E582, 2007

50. Park YS, Min KT, Kim TG, Lee YH, Cheong HJ, Yeom IS, et al. : Age-specific eNOS polymorphisms in moyamoya disease. Childs Nerv Syst 27 : 1919-1926, 2011

51. Qian C, Yu X, Li J, Chen J, Wang L, Chen G : The efficacy of surgical treatment for the secondary prevention of stroke in symptomatic moyamoya disease : a meta-analysis. Medicine (Baltimore) 94 : e2218, 2015

52. Smith ER : Moyamoya biomarkers. J Korean Neurosurg Soc 57 : 415421,2015

53. Smith ER, Scott RM : Progression of disease in unilateral moyamoya syndrome. Neurosurg Focus 24 : E17, 2008

54. Sun H, Wilson C, Ozpinar A, Safavi-Abbasi S, Zhao Y, Nakaji P, et al. : Perioperative complications and long-term outcomes after bypasses in adults with moyamoya disease : a systematic review and meta-analysis. World Neurosurg 92 : 179-188, 2016

55. Suzuki J, Takaku A : Cerebrovascular "moyamoya" disease. Disease showing abnormal net-like vessels in base of brain. Arch Neurol 20 : 288-299, 1969

56. Tendler BE, Shoukri K, Malchoff C, MacGillivray D, Duckrow R, Talmadge T, et al. : Concurrence of Graves' disease and dysplastic cerebral blood vessels of the moyamoya variety. Thyroid $7: 625-629,1997$

57. Würtz P, Raiko JR, Magnussen CG, Soininen P, Kangas AJ, Tynkkynen T, et al. : High-throughput quantification of circulating metabolites improves prediction of subclinical atherosclerosis. Eur Heart J 33 : 23072316, 2012 